International Journal of Navigation and Port Research

Vol.29, No.7 pp. 603 609, 2005 (ISSN-1598-7701)

\title{
Study on the Development of an Optimal Hull Form
}

\author{
Hee-Jong Choi* $\cdot$ Gyoung-Woo Lee** $\cdot$ Soon-Dong Youn*** $\cdot H o-H w a n$ Chun**** \\ *Division of Ocean System Engineering, Mokpo National Maritime University, Mokpo 530-729, Korea \\ **Division of Ocean System Engineering, Mokpo National Maritime University, Mokpo 530-729, Korea \\ ***Division of Ocean System Engineering, Mokpo National Maritime University, Mokpo 530-729, Korea \\ *****Department of Naval Architecture \& Ocean Engineering, Pusan National University, Busan 609-735, Korea
}

\begin{abstract}
This paper presents the method for developing an optimum hull form with minimum wave resistance using SQP(sequential quadratic programming) as an optimization technique. The wave resistance is evaluated by a Rankine source panel method with non-linear free surface conditions and the ITTC 1957 friction line is used to predict the frictional resistance coefficient. The geometry of the hull surface is represented and modified using NURBS(Non-Uniform Rational B-Spline) surface patches. To verify the validity of the developed program the numerical calculations for Wigley hull and Series $60 \mathrm{Cb}=0.6$ hull are performed and the results obtained after the numerical calculations are compared with the initial hulls.
\end{abstract}

Key words : Hull-optimization, SQP(sequential quadratic programming), Potential flow, Wigley hull, Series $60 \mathrm{Cb}=0.6$ hull

\section{Introduction}

Since the development of the landmark thin ship theory of Michell(1898), considerable efforts to predict the ship resistance have been devoted toward the development of CFD(Computational Fluid Dynamics) techniques over a century. The robust and practical CFD technique has been developed in the past decades and it has been used as a replacement to the towing tank testing since a CFD technique has advantages compared with the towing tank testing in terms of cost reduction as well as quantities of detailed flow field information. Among CFD techniques, the potential flow panel method based on Rankine sources with nonlinear free surface boundary conditions is preferable in ship yards because of its simplicity and the merits of computational cost in spite of its negligence of viscosity.

With the development of aforementioned CFD techniques and the improvement of computer performance the more comprehensive research to automatically modify the shape of hull form giving less resistance by CFD based optimization technique has been only very recently performed and interest in the prediction of the optimal hull form at a given design constraint has been ever growing. Although some of these researches have given some encouraging results, there are still a number of non-trivial difficulties: selection of an appropriate objective function, choice of optimization scheme, geometric representation of hull surface and choice of a practical, robust CFD tool to evaluate the objective function and the large computational cost to estimate the objective function through the repeated calculations during the whole optimization process.

Recently many interesting papers on the hull form optimization from a resistance point of view have been published. Tahara et al.(1998) developed a ship optimization method using the RANSE solver and SQP algorithm and performed hull optimization for Model 5415 hull and showed the optimized hull form. Hino et al.(1998) also developed a ship optimization method using Navier Stokes solver and SQP algorithm. Peri et al.(2001) presented several new optimized bulb shapes for a tanker with the aid of the potential flow solver using linearized free surface conditions and three different optimization algorithm(Conjugate Gradient, Steepest Descent and SQP), showing a verification by comparing with the experiments. Markov et al.(2001) performed optimization for Series $60 \mathrm{Cb}=0.6$ hull and HTC (Hamburg Test Case) containership using a higher order Rankine source panel method to evaluate the wave resistance and DFP(unconstrained Davidson Fletcher

Powell) as an optimization method and used a B spline patch to approximate the hull surface during the optimization process. Choi et al.(2003) developed a ship optimization method using the potential flow panel method based on Rankine sources with nonlinear free surface

\footnotetext{
* Corresponding Author : Hee-Jong Choi, choihj1988@hanmail.net 010)5509-0005

** kwlee@mmu.ac.kr 061)240-7083

*** sdong@mmu,ac.kr 061)240-7058

**** chunahh@pusan.ac.kr 051)510-2341
} 
boundary conditions and SQP method as a optimization technique and performed for KCS(KRISO Container Ship) and Series $60 \mathrm{Cb}=0.6$ hull.

In this study, an optimization procedure for a ship with minimum total resistance is stated. To predict the total resistance as an objective function, the friction resistance is estimated using the ITTC 1957 model ship correlation line formula and the wave resistance is evaluated using a potential flow panel method based on Rankine sources with nonlinear free surface boundary conditions where the sinkage and trim of the ship are fully taken into account. The geometry of hull surface is represented and modified using non uniform B spline surface patches during the optimization process in which the $\mathrm{B}$ spline coefficients are considered as the design parameters for the optimization.

To verify the validity of the optimization procedure, numerical computations are carried out for the Wigley hull and the Series $60 \mathrm{Cb}=0.6$ hull and the results obtained from the numerical computations are compared between the initial hull and the optimal hull.

\section{Optimization Algorithm}

\subsection{General optimization problem}

The general optimization problem may be expressed in the following form:

Minimize:

\section{$f(x)$}

Subjected to:

$$
\begin{aligned}
& g_{j}(x)=0, \quad j=1, \cdots, m_{e} \\
& g_{j}(x) \geq 0, \quad j=m_{e}+1, \cdots, m \\
& x_{l} \leq x \leq x_{u}
\end{aligned}
$$

where $f$ is an objective function, $g$ constraint functions, $x$ the vector of the design variables, $x_{l}$ and $x_{u}$ lower and upper limit of $x \mathrm{~m}$ the number of constraint functions and $m_{e}$ the number of equality constraint functions.

\subsection{Nonlinear programming algorithm}

In the present study, equation (1) and (2) are solved by sequential quadratic programming algorithm, in which the equations are approximated in quadratic form:

Minimize:

$$
\frac{1}{2} d^{T} B d+\nabla f(x)^{T} d
$$

Subjected to:

$$
\begin{aligned}
& \nabla g_{j}(x)^{T} d+g_{j}(x)=0 \\
& \nabla g_{j}(x)^{T} d+g_{j}(x) \geq 0
\end{aligned}
$$

where $d$ is search direction vector and $B$ approximate Hessian matrix of the Lagrangian. During the optimization process optimum $d$ is determined and $x$ is updated by $x^{n+1}=x^{n}+d$ in each iteration (Vanderplaats, 1984).

\section{Objective Function and Evaluation}

\subsection{Potential-based panel method}

Define a Cartesian coordinates system fixed on the ship which moves with a constant speed $U$ and the $\mathrm{x}$ axis points downstream and the $z$ axis upwards. In this frame of reference the vessel is stationary experiencing an incoming uniform stream $U$ along the positive $\mathrm{x}$ direction.

The fluid is assumed incompressible and inviscid and the flow irrotational, governed by the velocity potential $\phi$ subject to Laplace's equation in the fluid domain.

$$
\nabla^{2} \phi=0
$$

Over the wetted part of the hull surface the velocity potential must satisfy the hull boundary condition of no flow normal to the surface.

$$
n \cdot \nabla \Phi=0
$$

where $n$ is the unit normal vector.

The radiation condition must be satisfied

$$
\nabla \Phi \rightarrow(U, 0,0) \text { as } x^{2}+y^{2}+z^{2} \rightarrow \infty
$$

On the free surface the kinematic and the dynamic conditions must be satisfied as stated in the following equations

$$
\begin{aligned}
& \Phi_{x} n_{x}+\Phi_{x} n_{x}-\Phi_{z}=0 \text { on } z=\mathrm{n} \\
& \mathrm{n}=\frac{1}{2 g}\left(U^{2}-\nabla \phi \cdot \nabla \phi\right)
\end{aligned}
$$

Since equation (9) and (10) are fully nonlinear equations, in this paper the iteration procedure is used to solve the free surface problem based on the Rankine source panel method (Choi et al., 2001 ; Raven, 1996).

Having obtained the velocity potential and hence the flow velocity, the pressure coefficient at each panel can be found using Bernoulli's equation

$C_{p}=1-\frac{\nabla \phi \cdot \nabla \phi}{U^{2}}-2 \frac{z}{F n^{2}}$ 
The wave resistance coefficient $C_{W}$ is hence given by the pressure integral over the wetted hull surface

$$
C_{W}=-\frac{\int_{S} C_{p} n_{x} d S}{S}
$$

The frictional resistance coefficient is given by the ITTC 1957 model ship correlation line formula.

$$
C_{F}=\frac{0.075}{\left(\log _{10} R n-2\right)^{2}}
$$

where $S$ is the wetted surface of the hull and local Reynolds number is used for each element, based on the local velocity.

Finally the total resistance coefficient is obtained as follows

$$
C_{T}=C_{W}+(1+k) C_{F}
$$

where the form factor $(1+k)$ is calculated using the empirical formula presented by Holtrop et al.(1984).

\section{Modification of Hull Geometry}

\subsection{B spline surface modeling}

In the implementation of an optimization procedure, it is essential to have an efficient algorithm for geometry modification. The modified geometry should meet original design requirements without discontinuities in the surface and should be generally as smooth as possible. For ship hull optimization, it is necessary to use factors which are related to hull geometry generation as design variables. In this case, the increase of design variables means the increase of computation time. If the number of design variables is too small, realistic geometry may not be obtained. These requirements have been obtained by using a B spline patch:

$$
Q(u, v)=\sum_{i=1}^{n_{i}+1} \sum_{j=1}^{n_{j}+1} B_{i, j} N_{i, k}(u) M_{j, l}(v)
$$

where $B_{i, j}$ are the vertices of a defining polygon net, $N_{i, k}(u)$ and $M_{j, \lambda}(v)$ the $\mathrm{B}$ spline basis function in the bi parametric $u$ and $v$ directions, respectively.

The definition for the basis functions is as follows

$$
\begin{aligned}
& N_{i, 1}(u)= \begin{cases}1 & \text { if } \quad x_{i} \leq u \leq x_{i+1} \\
0 & \text { otherwise }\end{cases} \\
& N_{i, k}(u)=\frac{\left(u-x_{i}\right) N_{i, k-1}(u)}{x_{i+k-1}-x_{i}}+\frac{\left(x_{i+k}-u\right) N_{i+1, k-1}(u)}{x_{i+k}-x_{i+1}}
\end{aligned}
$$

and

$$
\begin{aligned}
& M_{j, 1}(v)= \begin{cases}1 & \text { if } \quad y_{j} \leq v \leq y_{j+1} \\
0 & \text { otherwise }\end{cases} \\
& M_{j, l}(v)=\frac{\left(v-y_{j}\right) M_{j, l-1}(v)}{y_{j+l-1}-y_{j}}+\frac{\left(y_{j+1}-v\right) M_{j+1, l-1}(v)}{y_{j+l}-y_{j+1}}
\end{aligned}
$$

where the $x_{i}$ and $y_{j}$ are elements of knot vectors.

\subsection{B spline surface fitting}

When the surface is described by external data, it is convenient to obtain an initial non flat B spline surface approximating the hull for subsequent real time interactive modification. This requires determining the defining polygon net from an existing network of three dimensional surface data points.

For each known surface data point equation (15) provides a linear equation in the unknown $B_{i, j}$ and similarly for all the surface data points. In matrix notation this can be written as

$$
[D]=[C][B]
$$

where $\quad C_{i, j}=N_{i, k} M_{j, t}$ For arbitrary $r_{s}$ topologically rectangular surface point data, $[D]$ is an $r * s \times 3$ matrix containing the three dimensional coordinates of the surface point data, [C] is an $r * s \times n * m$ matrix of the products of the B spline basis functions, and [B] is an $n * m \times 3$ matrix of the three dimensional coordinates of the required polygon net points.

Since for any arbitrary $*_{s}$ topologically rectangular surface point data, $[C]$ is not normally square, a solution can only be obtained in some mean sense. In particular

$$
[B]=\left[[C]^{T}[C]\right]^{-1}[C]^{T}[D]
$$

The $u$ and $v$ parametric values for each surface data are obtained using a chord length approximation (Huang el al., 1998 ; Rogers, 1990)

\section{Numerical Results}

Numerical analysis had been performed using the Wigley hull and the Series $60 \mathrm{Cb}=0.6$ hull to verify the validity of the hull optimization program which had been developed for this study. The Wigley hull and the Series $60 \mathrm{Cb}=0.6$ hull are commonly used for code validation and verification.

The Wigley hull could be assumed a comparatively thin ship. Therefore the flow around the hull could be regarded as potential flow. But in the case of Series $60 \mathrm{Cb}=0.6$ hull 
the hull form except near the bow is similar to a conventional container ship. Therefore the flow around the hull except the near bow is approximate to viscous flow while the flow near bow could be assumed potential flow. In this study the potential flow analysis technique had been used to solve the flow around a ship. Therefore the Wigley hull had been optimized throughout the whole hull surface while the Series $60 \mathrm{Cb}=0.6$ hull had been done in just local region in which potential flow is dominant.

\subsection{Wigley hull}

The Wigley hull is a notional hull whose shape is determined from its mathematical definition, which is given by

$$
y(x, z)=\frac{2}{B}\left(1-4 x^{2}\right)\left(1-\left(\frac{z}{D}\right)^{2}\right)
$$

where $B$ is the maximum thickness of the hull and D is the draft of the hull, and are set to 1 and 0.0625 respectively.
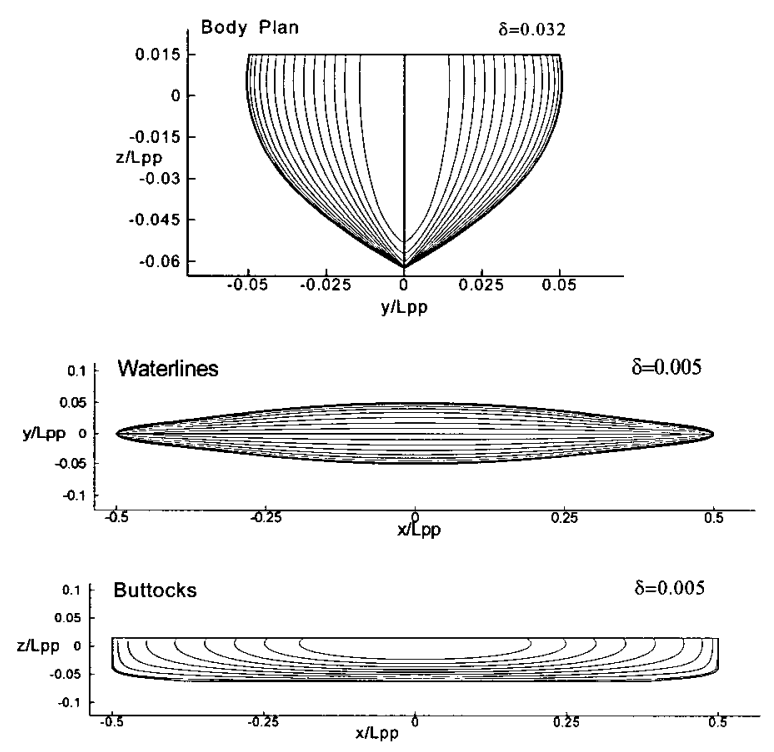

Fig. 1 Lines for the Modified Wigley hull taken as initial hull in the optimization

During the hull optimization procedure the hull is modified according to changes of the design variables and after the hull modification the hull should have reasonable hull shape in ship designer's point of view. Therefore to achieve the reasonable hull shape it had been known critical to keep $\mathrm{C} 1$ continuity of the hull geometry across the $y=0$. However the hull defined in equation (22) has discontinuity in gradient across the $y=0$ plane. Therefore the hull had been modified to keep $\mathrm{C} 1$ continuity across the $y=0$ plane.

The modified Wigley hull used as a initial hull for the hull optimization is shown in Fig. 1.

\section{Design variables}

Design variables for the Wigley hull had been achieved using B-spline surface fitting technique. $5 \times 9(5$ control vertices for each of 9 sections) control vertices network had been used to modify the hull.

The control vertices used for the hull modification had been automatically controlled to keep reasonable hull shape during the whole optimization procedure as shown in Fig. 2. The control vertices represented by $\diamond$ and $\square$ had been allowed to move in $\mathrm{x}^{-}$and $\mathrm{y}^{- \text {direction respectively and }}$ to move in both $\mathrm{x}^{-}$and $\mathrm{y}^{- \text {direction }}$ and in both $\mathrm{x}^{-}$and $z$-direction and $\mathbf{C}$ can move any direction and $\bigcirc$ had been allowed to move according to the neighbour control vertices. 1st and 2nd sections from the bow had been set to have the same $\mathrm{x}$-coordinate to keep $\mathrm{Cl}$ continuity across the $y=0$ plane and 8th and 9th sections from the stern to have the same $\mathrm{x}$-coordinates.

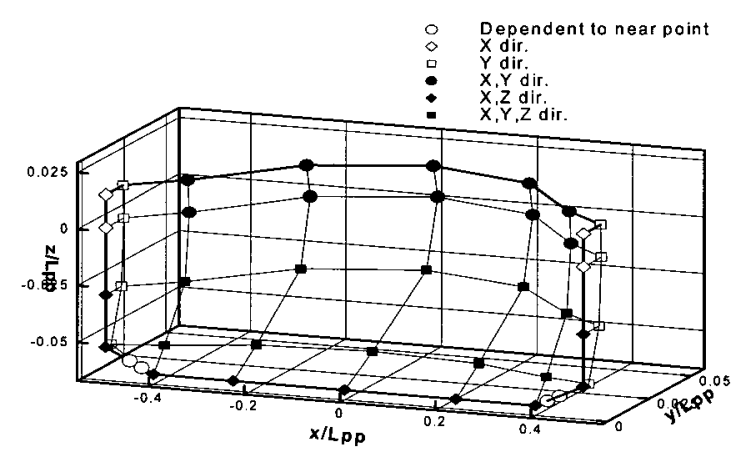

Fig. 2 Design variables for the Wigley hull taken as initial hull

The movable limitations of the design variables had been given by

$$
\begin{aligned}
-0.700 & <x / L p p<0.700 \\
0.000 & <y / L p p<0.080 \\
-0.080 & <z / L p p<-0.010
\end{aligned}
$$

\section{Constraint conditions}

In the ship designer's point of view the displacement is most important consideration and during the hull optimization procedure the displacement of the modified hull should be preserved as the same or greater value than the initial hull therefore. In this study the displacement had been confined as the same value of the initial hull and the waterplane transverse moment of inertia had also been used as a equality constraint condition and to keep ship-like hull form the $y$-component of the normal vector on the hull surface should be the same or greater than 0 and 
formulated as

$n_{y} \geq 0$

Numerical Results

The hull optimization had been performed at design speed of $\mathrm{Fn}=0.316$ and the total resistance as an objective function had been obtained using the potential-based panel method in which the non-linearities of the free surface had been considered and ITTC 1957 model-ship correlation formula.

Fig. 3 shows the lines of the optimal hull. The optimal hull has the bulbous form both at the bow and at the stern, and also has ship like stern type.
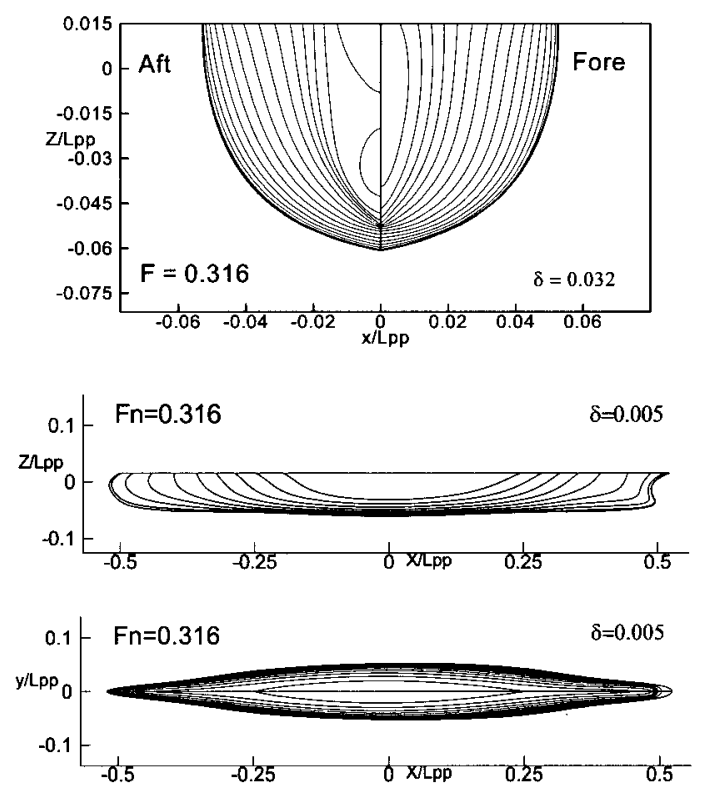

Fig. 3 Lines for optimized hull at $F n=0.316$

Fig. 4 shows the comparison of the wave system between the initial hull and the optimal hull. The wave induced by the optimal hull is greatly reduced compared to the one of the initial hull and especially the wave generated from the shoulder is conspicuously decreased due to the fact of the existence of the bulbous bow of the optimal hull.

In Fig. 5 the wave heights along the hull side wall are compared.

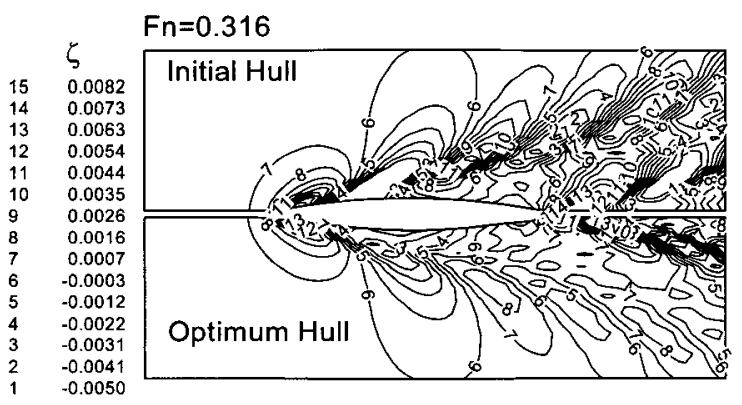

Fig. 4 Comparison of wave patterns induced by the initial hull and the optimal hull optimized at Fn=0.316

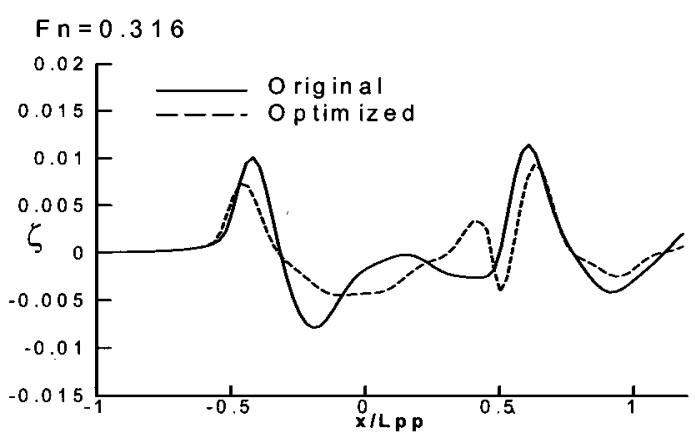

Fig. 5 Comparison of wave height along the hull side between the initial hull and the optimal hull.

Table 1 Comparison of the hydrodynamic data between the initial hull and the optimal hull.

\begin{tabular}{|c|c|r|r||}
\hline & \multicolumn{1}{|c|}{ Initial } & \multicolumn{1}{c|}{ Optimal } & $\Delta(\%)$ \\
\hline$R_{W M}$ & 0.11600 & 0.02310 & -80.09 \\
\hline$R_{T M}$ & 0.48860 & 0.37340 & -23.58 \\
\hline
\end{tabular}

In Table 1 the hydrodynamic data are compared between the initial hull and the optimal hull. The total resistance used as a objective function is reduced about $24 \%$ in the optimal hull compared to the one of the initial hull and especially in the wave resistance point of view the efficiency of the optimal hull is dramatically improved since the objective function had been estimated using the potential-based panel method in which the frictional resistance of the ship is just dependent upon the wetted hull surface area.

\subsection{Series $60 \mathrm{Cb}=0.6$ Hull}

Series $60 \mathrm{Cb}=0.6$ hull had been optimized using the developed hull optimization technique. The region near the bow of the ship had been modified and optimized during the whole hull optimization procedure and the other region of the ship had been fixed.

\section{Design variables}

The hull optimization for Series $60 \mathrm{Cb}=0.6$ hull had been confined to just $25 \%$ hull surface from the bow since in the last $75 \%$ hull surface the flow could not be assumed potential flow and the viscous flow is more dominant therefore during the optimization procedure this region had not been allowed to move. Control vertices had been generated using $\mathrm{B}$-spline surface fitting technique as shown in Fig. 6 and the control vertices represented by 0 had been allowed to move in just $\mathrm{y}$-direction and the control vertices by $\mathrm{X}$ in just $\mathrm{x}$-direction. 


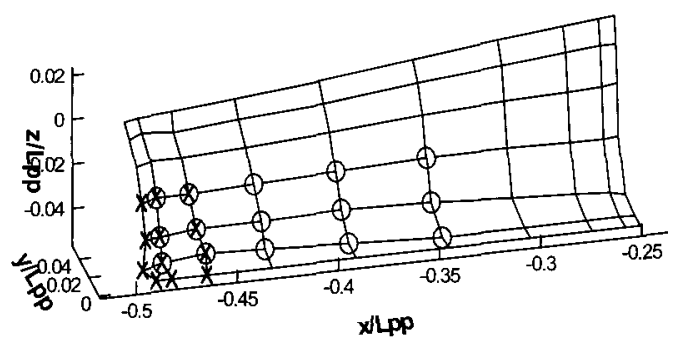

Fig. 6 Design Variables for Series $60 \mathrm{Cb}=0.6$ Hull

In ship design the area of the waterplane is an important consideration. Therefore the control vertices below the waterline had been used as a design variables. During the hull optimization procedure the $\mathrm{C} 1$ continuity of the hull between the movable and the fixed region of the ship surface had been preserved as the control vertices after 6 -th section had been let fixed.

The movable limitations of the design variables had been given by

$$
\begin{aligned}
& -0.5300<x / L<-0.480 \\
& 0.0000<y / L<0.050 \\
& -0.0535<z / L<0.000
\end{aligned}
$$

\section{Constraint Conditions}

In general the displacement of the hull is set fixed in hull optimization. However in this study the region near the bow had been considered as a optimization region. Therefore if the displacement of the hull is fixed then the variation of the objective function is too small. Therefore in this study the displacement of the hull had been set equal or greater than the initial hull during the optimization procedure.

To keep the reasonable hull shape during the hull optimization procedure it is recommendable to apply the condition written in equation (26)

$$
0 \leq \theta \leq 90^{\circ}
$$

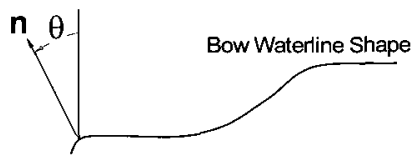

Fig. 7 Definition of $\Theta$

\section{Numerical Results}

The Series $60 \mathrm{Cb}=0.6$ hull had been optimized at the design speed of $\mathrm{Fn}=0.316$ and non-linear free surface boundary conditions had been applied to satisfy the free surface boundary condition in the potential flow analysis.

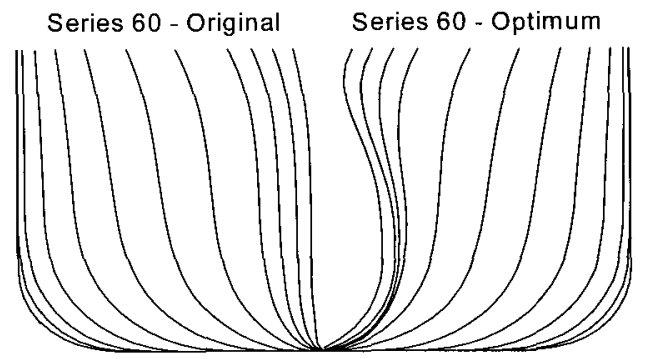

Fig. 8 Comparison of Body Plan

Fig. 8 shows the comparison of the body plan between the initial hull and the optimal hull obtained through the hull optimization procedure. As shown in the figure the optimal hull has the big bulbous bow compared to the one of the initial hull and during the hull optimization at each hull modification it had been known that the hull surface had been modified in smooth way in the ship designer's point of view since the condition in equation (26) had been enforced in hull modification.

In Fig. 9 and Fig. 10 the wave pattern and the wave height along the ship side of the optimal hull are compared with the ones of the initial hull. The wave is greatly reduced and especially the wave generated from the shoulder of the hull is conspicuously decreased due to the fact of the existence of the bulbous bow.

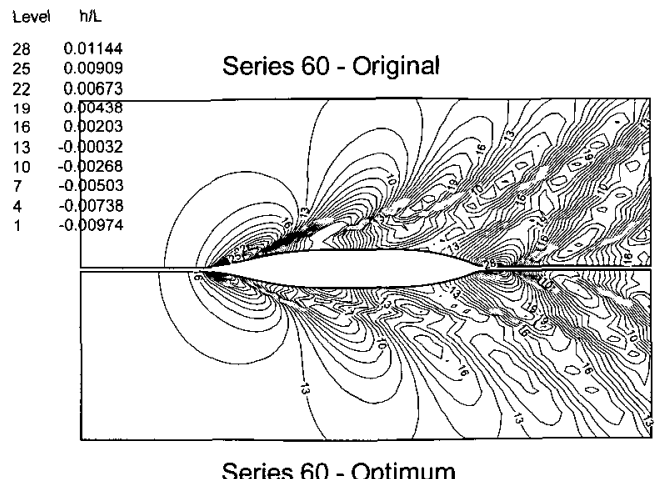

Fig. 9 Comparison of Wave Contour

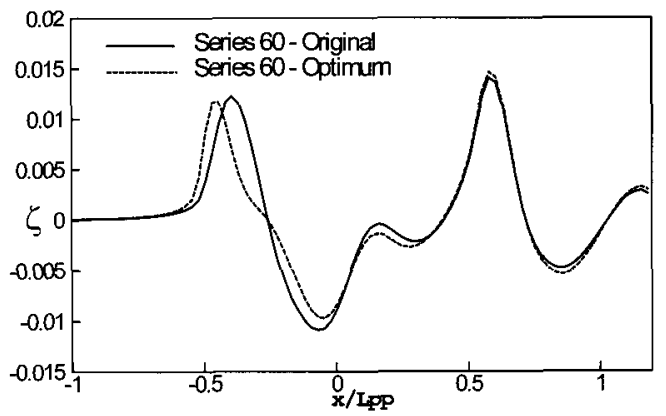

Fig. 10 Comparison of Wave Profile 
Table 2 hydrostatic and hydrodynamic data

\begin{tabular}{cccc}
\hline \hline & Initial & Optimal & \multicolumn{1}{c}{$\Delta(\%)$} \\
\hline$C_{B}$ & 0.5976 & 0.6073 & +1.6230 \\
$S_{\text {wet }}$ & 0.1706 & 0.1719 & +0.7620 \\
$R_{W M}$ & 0.1324 & 0.0935 & -29.3810 \\
$R_{T M}$ & 0.5477 & 0.5117 & -6.5730 \\
\hline \hline
\end{tabular}

Table 2 compared the hydrostatic and hydrodynamic characteristics between the initial and the optimal hull. As mentioned the ITTC 1957 model-ship correlation formula had been used to achieve the total resistance and 1.029 had been used as form factor $(1+k)$. As shown in the Table. 2 the displacement and the wetted hull surface area had been a little increased however the total resistance had been reduced $6.57 \%$ and the wave making resistance had been decreased $29.38 \%$.

\section{Conclusion}

Hull optimization program had been developed using the optimization algorithm and the potential flow analysis technique with nonlinear free surface conditions. To verify the validity of the developed numerical program numerical computations had been performed for the Wigley hull and Series $60 \mathrm{Cb}=0.6$ hull in design speed of $\mathrm{Fn}=0.316$.

The numerical results had been shown

1. The wave system induced by the optimal hull had been conspicuously reduced compared to the one induced by the initial hull.

2. Total resistance or wave making resistance had been greatly reduced during the hull optimization procedure in spite of the increase of the displacement and the wetted hull surface area.

3. The bulbous bow shape had been generated as a result of the hull optimization and proven as an efficient installation in the resistance point of view.

\section{Reference}

[1] Choi H. J., Chun H. H.(2001), "Potential Flow Analysis for a Hull with the Transom Stem", J. of KCORE, Vol 15, No 1, pp. 1-6(in Korean).
[2] Choi H. J., Seo K. C., Kim B. E. and Chun H. H.(2003), "Development of an Optimum Hull Form for a Container Ship with Minimum Wave Resistance", J. of SNAK, Vol 40, No 5, pp.8-15(in Korean).

[3] Hino, T., Kodama, Y. and Hirata, N.(1998), "Hydrodynamic Shape Optimization of Ship Hull Forms Using CFD", Proceedings 3rd Osaka Colloquium on Advanced CFD Applications to Ship Flow And Hull Form Design, Osaka, Japan, May 25-27, pp. 533 -541.

[4] Holtrop, I. J.(1984), “A Statistical Re-analysis of Resistance and Propulsion Data”, International ShipBuilding Progress, Vol 31. pp. 272 276.

[5] Huang, C. H., Chiang, C. C. and Chou, S. K.(1998), "An Inverse Geometry Design Problem in Optimizing Hull Surfaces", J. of Ship Research, Vol 42, No 20, pp. 79-85.

[6] Markov, N. E., Suzuki, K.(2001), "Hull Form Optimization by Shift and Deformation of Ship Sections", J. of Ship Research, Vol 45, No 3, pp. 197-204.

[7] Peri, D., Michele, R. and Emilio, F. C.(2001), "Design Optimization of Ship Hulls via CFD Techniques", Journal of Ship Research, Vol 45, No 2, pp. 140-149.

[8] Raven, H. C.(1996), "A Solution Method for the Nonlinear Ship Wave Resistance Problem", Doctor's Thesis, Delft Univ. Techn., Delft, Netherlands.

[9] Rogers, D. F. and Adams, J. A.(1990), "Mathematical Elements for Computer Graphics", McGraw-Hill, New York.

[10] Tahara, Y., Himeno, Y. and Tsukahara, T.(1998), “An Application of Computational Fluid Dynamics to Tanker Hull Form Optimization Problem", Proceedings 3rd Osaka Colloquium on Advanced CFD Applications to Ship Flow And Hull Form Design, Osaka, Japan, May 25-27, pp. 515 531.

[11] Vanderplaats, G. N.(1984), "Numerical Optimization Techniques for Engineering Designs”, McGrow-Hill, New York.

Received 27 June 2005

Accepted 26 September 2005 